
\title{
28 Research Square \\ Vaccination for Patients with Inborn Errors of Immunity: A Nationwide Survey in Japan
}

\section{Sho Hosaka ( $\nabla$ shohosaka@md.tsukuba.ac.jp )}

University of Tsukuba Hospital https://orcid.org/0000-0002-2792-1235

\section{Takahiro Kido}

University of Tsukuba Hospital

\section{Kazuo Imagawa}

University of Tsukuba Hospital

Hiroko Fukushima

University of Tsukuba Hospital

\section{Tomohiro Morio}

Tokyo Medical and Dental University Graduate School

\section{Shigeaki Nonoyama}

National Defense Medical College

Hidetoshi Takada

University of Tsukuba Hospital https://orcid.org/0000-0002-0741-5060

\section{Research Article}

Keywords: Inborn errors of immunity, epidemiology, Japan, vaccine, BCG

Posted Date: July 16th, 2021

DOl: https://doi.org/10.21203/rs.3.rs-632171/v1

License: (c) (i) This work is licensed under a Creative Commons Attribution 4.0 International License.

Read Full License

Version of Record: A version of this preprint was published at Journal of Clinical Immunology on October 27th, 2021. See the published version at https://doi.org/10.1007/s10875-021-01160-x. 


\section{Abstract}

We conducted a nationwide survey of inborn errors of immunity (IEI) in Japan for the second time in 10 years, focusing on protective measures for IEl patients against infectious diseases. Questionnaires were sent to various medical departments nationwide, and a total of 1,307 patients were reported. The prevalence of IEI was 2.2 patients per 100,000 population which was comparable with the previous nationwide study. The most common disease category was autoinflammatory disorders (25\%), followed by antibody deficiencies (24\%) and congenital defects of phagocyte number or function (16\%). We found that a significant number of patients received contraindicated vaccines, principally because the patients were not diagnosed as IEl by the time of the vaccination. Regarding diseases for which BCG vaccination is contraindicated, $43 \%$ patients had actually received BCG, of which $14 \%$ developed $B C G$ related infections. In order to prevent IEI patients from receiving inadequate vaccines, the continuous education to parents and physicians is needed, along with the expansion of newborn screening, but efforts to screen IEl at the site of vaccination also remains important.

\section{Introduction}

The majority of patients with inborn errors of immunity (IEI) are susceptive to various types of infections, emphasizing the importance of infection prophylaxis in this population. Vaccines are important for establishing immunity against pathogens, but they may be contraindicated in a subgroup of IEl patients due to potential severe vaccine-related adverse events, especially infections due to live vaccine strains [1]. Since children receive routine vaccine administration beginning as early as several months of age, it is important to diagnose IEl early enough to prevent any serious vaccine strain infections. However, the actual proportion of patients who receive contraindicated vaccines before diagnosis in Japan is unknown. Childhood vaccine protocols varies between countries due to differences in vaccine cost or insurance policies, and the epidemic status of pathogens. One example is the Bacille de Calmette et Guérin (BCG) vaccine, which is not routinely administered in many Western countries, but is part of a routine schedule in Japan, where tuberculosis is an endemic disease [2]. This indicates that Japanese IEI patients are at a high risk for BCG-related infections, but although the prevalence of BCG infections has been reported in various countries [3,4], the situation for Japanese IEI patients is unknown. Furthermore, concerns regarding appropriate immunization for IEl patients are increasing, since the number of newly diagnosed IEI patients is expanding due to advances in diagnostic methods such as next generation sequencing (NGS) [5]. In 2018, we conducted a nationwide survey of IEI patients, focusing on protective measures against infectious diseases. The present study focuses on results from the survey regarding individual vaccination status in Japanese IEI patients, aiming to elucidate the situation of pre-diagnostic vaccination. Regrading BCG, we also analyzed the rate of BCG-related adverse events after inoculation.

\section{Methods}

This study was conducted according to the nationwide epidemiological survey manual of patients with intractable diseases (3rd edition, 2017), as was the previous survey which was published in 2011 [6]. Five 
clinical departments (pediatrics, internal medicine, hematology, rheumatology, dermatology) were selected for having a high tendency of attending to IEl patients. A primary questionnaire requesting the number of IEl patients treated between January 1, 2018 and December 31, 2018 was sent to $20 \%$ of all of the five departments in hospitals nationwide; hospitals were selected randomly after setting selection ratios according to hospital size and academic status (table 1, table $S 1$;Online Resource 1 ). A secondary survey was performed by sending questionnaires to departments where at least one patient was treated during the observation period. The secondary questionnaire was comprised of the following questions; 1 ) general information concerning age, sex, and diagnosis, 2) clinical symptoms or signs leading to diagnosis, 3) a description of all treatment (eg, IgG replacement, antibiotic prophylaxis, stem cell transplantation (SCT), etc), 4) a description of all previous vaccines, and 5) the vaccination policy of the attending physician. We classified diseases according to the International Union of Immunological Societies (IUIS) 2017 classifications [5]. Statistical analysis was performed using GraphPad Prism software version 8.0 (San Diego, CA, USA). The protocol for this study was approved by the ethics committee at University of Tsukuba Hospital (H30-208).

\section{Results}

A primary survey was performed by sending questionnaires to 12,517 departments nationwide. Response rates varied between departments, from $18 \%$ (internal medicine) to $55 \%$ (pediatrics) with an average of $28 \%$. This was comparable to the previous nationwide survey where the response rate for internal medicine and pediatrics was $20 \%$ and $55 \%$, respectively [6]. A total of 1,307 patients were reported from 706 departments. The estimated number of IEl patients in Japan was 2,794 (95\% confidence interval 2,334-3,019) (table 1). The population of Japan in 2018 was 126 million [7], giving a prevalence of 2.2 IEI patients per 100,000 population. Detailed information from secondary questionnaires was obtained for 750 patients (response rate $57 \%$ ), and the rate of any genetic diagnosis was $74 \%$ (data not shown). The reported patients are listed in table 2 . The most common IEl category was autoinflammatory disorders $(n=191,25 \%)$, followed by predominantly antibody deficiencies $(n=183,24 \%)$ and congenital defects of phagocyte number or function $(n=121,16 \%)$. Compared to the previous survey in 2011 [6], we observed an increase in autoinflammatory disorders and defects in intrinsic and innate immunity, and a relative decrease in other categories. The male to female ratio was 1.46:1 ( $n=744$, unanswered: 6 cases). The patient's median age at the time of study was 18.0 years (range: 0 to 85.4 years), and the median age at diagnosis was 2.0 years (range: 0 to 79 years). The distribution of diagnostic age for each disease category is shown in figure 1. The median age at diagnosis for patients with autoinflammatory disorders (7.1 years), complement deficiencies (22.3 years), and phenocopies of IEI (21.0 years) was significantly higher than other groups (figure 1).

The actual vaccination history for each patient was acquired and analyzed. Patients were grouped into either of the following; 1) patients who received BCG, 2) patients with a positive history of a live vaccine but no BCG, or 3) patients who received inactivated vaccines only. Regarding patients who received SCT, only vaccines that were administered before SCT were counted, since these patients are mostly rescheduled for regular vaccines after SCT. We counted pre-SCT vaccines under the assumption that 
vaccines were administered according to routine schedules in Japan [8]. Patients with insufficient data concerning vaccination or SCT status were removed $(n=9)$. Since one of our objectives was to investigate the rate of pre-diagnostic vaccines, we also excluded patients with autoinflammatory disorders, complement deficiencies, and phenocopies of IEI because they were mostly diagnosed at an age later than the period for routine vaccination in childhood (figure 1). The individual vaccination history for several representative diseases within each IUIS category is shown in figure 2. While live vaccines are generally contraindicated in patients with severe combined immunodeficiency (SCID) or combined immunodeficiency (CID) [1], we observed that $26 \%$ of SCID patients and $54 \%$ of CID patients had received some form of live vaccine. Likewise, we saw that live vaccines were administered to $56 \%$ of patients with anhidrotic ectodermal dysplasia with immunodeficiency (EDA-ID) and $36 \%$ of patients with familial hemophagocytic lymphohistiocytosis (FHL), for whom live vaccines are thought to be harmful $[9,10]$. Regarding patients with chronic granulomatous disease (CGD) or mendelian susceptibility for mycobacterial disease (MSMD), for whom BCG is generally contraindicated [11], the rate of patients who actually received $B C G$ was $48 \%$ and $82 \%$ for CGD and MSMD, respectively. The rate of patients with hyper IgE syndrome (HIES) and chronic mucocutaneous candidiasis (CMC) who received BCG was $39 \%$ and $50 \%$, respectively. Of note, $97 \%$ of HIES patients were diagnosed with STAT3 deficiency, and $100 \%$ of patients with CMC were diagnosed with STAT1 gain of function (GOF) mutations. The vaccination history for patients with other diseases are shown in figure S1 (Online Resource).

We retrieved information concerning any history of BCG related infections (BCG-I). We selected diseases for which BCG is generally contraindicated (SCID, CID, CGD, MSMD), or diseases with reports of severe BCG infection (EDA-ID, STAT3 deficiency, STAT1 GOF) $[3,12,13]$. The results for all pooled patients, and for each disease are shown in figure 3. The number of patients who received BCG for the pooled patients was $83(43 \%)$, of which 27 (14\%) had a reported BCG-I. When focusing on individual diseases, we saw that there was no reported BCG-I for SCID and CID, while the prevalence of BCG-I was high among CGD $(n=20,25 \%)$ and MSMD $(n=6,55 \%)$. We also observed one report case of BCG-I among EDA-ID $(n=1$, $11 \%)$. All cases of BCG-I from the whole survey are summarized in Table 3. Among other diseases, there were cases of BCG-I among patients with WAS/XLT (n=2), and XLP $(n=1)$. Other than BCG-I, there were two reported cases of rotavirus-vaccine related enterocolitis in patients with SCID, and one case of vaccine-derived varicella zoster in a patient with EDA-ID.

Regarding vaccination policies for each patient, physicians were questioned whether their policy was to 1) administer all vaccines, 2 ) administer all vaccines except BCG, 3) allow inactivated vaccines only, or 4) allow no vaccines. For this analysis, we excluded patients who received SCT, since vaccination policy differs greatly with SCT status. There were no cases of SCID for which the policy was to administer live vaccines (figure 4). The policy was to avoid all vaccines in $68 \%$ of XLA cases, possibly reflecting the fact that most patients were on IgG replacement therapy. Regarding BCG administration, the policy to avoid BCG was seen in $70 \%$ and $73 \%$ of CGD and MSMD cases, respectively.

\section{Discussion}


We conducted a nationwide survey of IEI for the second time in 10 years. The prevalence of IEI was 2.2 per 100,000 inhabitants, which was comparable to the previous nationwide study $(2.3 / 100,000)$ [6]. We observed a marked increase in the percentage of patients with autoinflammatory disorders, which was the most prevalent disease category in this study. This increase possibly reflects advances in genetic testing and a better understanding for this disease group, since autoinflammatory disease was introduced into the spectrum of IEI rather recently [14]. The relative decrease in 'classical' IEI such as CID or antibody deficiencies compared to the previous study may be due to a difference in the selection rate of pediatric departments. We set the selection rate to $20 \%$ for all medical departments following the epidemiological survey manual [15], which likely shifted our cohort to an older age compared with previous studies which mainly selected pediatric patients $[6,16,17]$. This is supported by the fact that the proportion of patients reported from pediatric departments was $77 \%$ (table 1 ), compared with $92 \%$ from the previous study [6]. The older age of our cohort may also be due to advances in diagnoses and management, as more patients with IEl grow into adulthood and more adults are newly diagnosed with IEI [18].

A genetic diagnosis was made in $74 \%$ of patients, which is relatively high compared to other studies [19], and is again attributable to advances in diagnostic methods, such as the use of next-generation sequencing. We observed a difference in diagnostic age between disease groups (Fig. 1), which is in line with previous studies $[20,21]$. Another interesting finding was the disease distribution within antibody deficiencies; compared with the previous study, we observed an increase in CVID which outnumbered $\mathrm{XLA}$. Although CVID is a heterogenous disease and many patients lack a genetic diagnosis, our results may reflect a growing recognition of IEl among non-pediatric physicians, since many patients with CVID are diagnosed in adulthood [22].

One of the objectives of this survey was to understand the current status of vaccination in patients with IEI in Japan. We observed that a significant percentage of patients received a contraindicated vaccine, presumably before diagnosis of the disease. For example, $26 \%$ of SCID and $54 \%$ of CID patients had received a live vaccine (Fig. 2), which is contraindicated in this setting. We should assume that these patients may have developed serious adverse events, because patients with these diseases are known to have an increased risk when receiving live vaccines [23]. Likewise, the proportion of patients with SCID, CGD, and MSMD patients who received BCG was $23 \%, 48 \%$, and $82 \%$, respectively.

Regarding the issue of BCG vaccination, physicians were asked to report any history of BCG related infections since they could be important signs of IEI. We saw that within the 195 patients who received an 'undesirable' BCG, 27 patients suffered from BCG related infections, which may be fatal in patients with IEI $[24,25]$ (Fig. 3). When focusing on individual diseases, we saw that most of the reported BCG infections were from patients with CGD or MSMD, and that there were no reports from patients with SCID or CID. This is in contrast with a previously published review of BCG vaccination in SCID patients, where $51 \%$ of patients developed BCG complications [25]. However, most of the patients described in the review received BCG during the first month of life, whereas Japanese patients typically receive BCG during 5 to 7 months of age [8]. Since most SCID patients receive SCT, the patients from our cohort may have cured 
before any severe manifestations of BCG infection. Another interesting finding was the report of BCG infection in XLP (Table 3), since there have been no previous reports of BCG infection with this disease, and XLP is rather known for overproduction of interferon gamma, which is important for the eradication of mycobacterium species.

The role of BCG vaccines in preventing tuberculosis is unquestionable, especially for the prevention of meningitis and miliary tuberculosis in infants [26]. However, like other vaccines, the policy of vaccination depends on the prevalence of the pathogen. In western Europe or USA, where the prevalence of tuberculosis is low, BCG vaccination is reserved for special high risk groups [2]. The incidence of tuberculosis in Japan has decreased greatly since the 1950's [27]. However, the incidence among youngaged people shows a slight increase during the recent period [28], and Japan is still considered a moderately tuberculosis endemic country. This suggests the continuous need for universal BCG vaccination in Japan, along with the need of diagnosing at-risk patients before BCG.

Newborn screening (NBS) for SCID has proven efficacy in detecting immunodeficient patients before clinical manifestation, and is adopted as a universal program in several countries such as USA [29], Israel [30], and Spain [31]. Although SCID-NBS is only available in limited areas in Japan [32], if it can be applied on a national scale, it may become one solution to the problem of inappropriate vaccination in pre-diagnosed patients. In addition, efforts should be focused on screening patients with CGD and MSMD at the site of vaccination, until novel NBS methods become available [12,33]. For example, physicians, especially pediatricians, should ask specific questions about the patient's condition and family history; asking about any family history of IEI may be insufficient, and a more specific interview regarding a family history of severe BCG-I or childhood disseminated tuberculosis infection may be necessary [34,35].

One limitation of this study is that it was based on physician directed questionnaires making it subject to reporting bias; an absence of documented adverse events may not necessarily mean there were no actual events. Another important fact is that this was a cross-sectional study, and included patients diagnosed at various ages, some of whom were diagnosed more than 50 years ago. Although we assessed vaccine indications using present guidelines for this study, evidence surrounding IEI has obviously changed over the past years. For example, patients with chronic mucocutaneous candidiasis (CMC) were generally thought to have immunity against pathogens other than candida species, but there is emerging evidence that patients with STAT1 GOF mutations, the most common form of CMC, display susceptibility against various pathogens including the BCG strain [36]. Therefore, patients who received seemingly inappropriate vaccines in the past according to present guidelines, may have been vaccinated following up-to-date evidence at that time. Nevertheless, no registry concerning vaccination status and diagnosis exists in Japan, so we believe the results of this study serves as valuable evidence in this field currently available.

For this study, we categorized patients according to recent classifications [5], but one must note that immunological profiles may differ even among patients with the same disease. For example, defects in the IL2RG gene typically leads to T + B-SCID, but there are increasing reports of hypomorphic IL2RG 
mutations leading to a less severe or 'leaky' phenotype, making early diagnosis difficult [37]. Therefore, a thorough immunological survey of each patient is important, and physicians are recommended to consult a clinical immunologist before considering immunization for atypical cases [38].

\section{Conclusions}

In summary, we conducted a national survey of IEl in Japan for the second time in 10 years, and observed an increase in diseases that are well diagnosed in adulthood. A significant number of patients had received contraindicated vaccines before diagnosis, underscoring the need for continuous education to parents and physicians. The expansion of NBS is expected, but efforts to screen IEI at the site of vaccination continues to be important.

\section{Declarations}

\section{Acknowledgements}

The authors thank all the physicians who participated in this survey, and Ms. Hirosawa for clerical assistance. This study was funded by Health and Labour Science Research Grants for Research on Intractable Diseases from the Ministry of Health, Labour and Welfare of Japan (17933299).

Funding: This study was funded by Health and Labour Science Research Grants for Research on Intractable Diseases from the Ministry of Health, Labour and Welfare of Japan (17933299).

Conflicts of interest: The authors declare that they have no conflict of interest.

Availability of data and material: The data analyzed in the current study is available from the corresponding author on reasonable request.

Code availability: Not applicable

Authors' contributions: Sho Hosaka and Takahiro Kido contributed to the analysis of the results and to the writing of the manuscript. Takahiro Kido and Hidetoshi Takada designed the study and performed the survey. Kazuo Imagawa, Hiroko Fukushima, Tomohiro Morio, Shigeaki Nonoyama, and Hidetoshi Takada revised the manuscript for important intellectual content. All authors commented on the paper and approved the final manuscript as submitted.

Ethics approval: The protocol for this study was approved by the ethics committee at University of Tsukuba Hospital (H30-208)

Consent to participate: Informed consent was obtained from the parents or their legal guardians in the form of opt-out on web-site. 
Consent for publication: Informed consent was obtained from the parents or their legal guardians in the form of opt-out on web-site.

\section{References}

1. Pöyhönen L, Bustamante J, Casanova JL, Jouanguy E, Zhang Q. Life-Threatening Infections Due to Live-Attenuated Vaccines: Early Manifestations of Inborn Errors of Immunity. J. Clin. Immunol. 2019;39(4):376-90.

2. Public Health Agency of Canada. The BCG World Atlas $3^{\text {rd }}$ edition, updated in 2020. http://www.bcgatlas.org/index.php 2020. Accessed $15^{\text {th }}$ May 2021.

3. Nunes-Santos CdJ, Rosenzweig SD. Bacille Calmette-Guerin Complications in Newly Described Primary Immunodeficiency Diseases: 2010-2017. Front. Immunol. 2018;9(1423).

4. Yadav RM, Dalvi A, Gupta M, Bargir UA, Shabrish S, Aluri J, et al. Spectrum of Inborn errors of immunity in a cohort of 90 patients presenting with complications to BCG vaccination in India. Scand. J. Immunol. 2021;93(5):e13010.

5. Picard C, Bobby Gaspar H, Al-Herz W, Bousfiha A, Casanova J-L, Chatila T, et al. International Union of Immunological Societies: 2017 Primary Immunodeficiency Diseases Committee Report on Inborn Errors of Immunity. J. Clin. Immunol. 2018;38(1):96-128.

6. Ishimura M, Takada H, Doi T, Imai K, Sasahara Y, Kanegane H, et al. Nationwide Survey of Patients with Primary Immunodeficiency Diseases in Japan. J. Clin. Immunol. 2011;31(6):968-76.

7. Statistics Bureau of Japan. Current Population Estimates as of October 1, 2018. http://www.stat.go.jp/data/jinsui/2018np/index.html. Accessed 15 ${ }^{\text {th }}$ May 2021.

8. Saitoh A, Okabe N. Progress and challenges for the Japanese immunization program: Beyond the "vaccine gap". Vaccine. 2018;36(30):4582-8.

9. Bonilla FA, Khan DA, Ballas ZK, Chinen J, Frank MM, Hsu JT, et al. Practice parameter for the diagnosis and management of primary immunodeficiency. J. Allergy Clin. Immunol. 2015;136(5):1186-205.e1-78.

10. Gholam C, Grigoriadou S, Gilmour KC, Gaspar HB. Familial haemophagocytic lymphohistiocytosis: advances in the genetic basis, diagnosis and management. Clin. Exp. Immunol. 2011;163(3):271-83.

11. Principi N, Esposito S. Vaccine use in primary immunodeficiency disorders. Vaccine. 2014;32(30):3725-31.

12. Fekrvand S, Yazdani R, Olbrich P, Gennery A, Rosenzweig SD, Condino-Neto A, et al. Primary Immunodeficiency Diseases and Bacillus Calmette-Guérin (BCG)-Vaccine-Derived Complications: A Systematic Review. J. Allergy Clin. Immunol. In Practice. 2020;8(4):1371-86.

13. Tsilifis C, Freeman AF, Gennery AR. STAT3 Hyper-IgE Syndrome-an Update and Unanswered Questions. J. Clin. Immunol. 2021 May 1. https://10.1007/s10875-021-01051-1, Online ahead of print. 
14. Notarangelo L, Casanova J-L, Fischer A, Puck J, Rosen F, Seger R, et al. Primary immunodeficiency diseases: An update. J. Allergy Clin. Immunol. 2004;114(3):677-87.

15. Nakamura Y. Nationwide Epidemiological Survey Manual of Patients with Intractable Diseases, 3rd ed. Research Committee on Epidemiology of Intractable Disease; 2017.

http://www.jichi.ac.jp/dph/nanbyou/manual_2017.pdf. Accessed 15 $5^{\text {th }}$ May 2021.

16. Kobrynski L, Powell RW, Bowen S. Prevalence and Morbidity of Primary Immunodeficiency Diseases, United States 2001-2007. J. Clin. Immunol. 2014;34(8):954-61.

17. Rhim JW, Kim KH, Kim DS, Kim BS, Kim JS, Kim CH, et al. Prevalence of primary immunodeficiency in Korea. J. Korean Med. Sci. 2012;27(7):788-93.

18. Rosenberg E, Dent PB, Denburg JA. Primary Immune Deficiencies in the Adult: A Previously Underrecognized Common Condition. J. Allergy Clin. Immunol. In Practice. 2016;4(6):1101-7.

19. Abolhassani H, Azizi G, Sharifi L, Yazdani R, Mohsenzadegan M, Delavari S, et al. Global systematic review of primary immunodeficiency registries. Expert. Rev. Clin. Immunol. 2020;16(7):717-32.

20. Mahlaoui N, Picard C, Bach P, Costes L, Courteille V, Ranohavimparany A, et al. Genetic diagnosis of primary immunodeficiencies: A survey of the French national registry. J. Allergy Clin. Immunol. 2019;143(4):1646-9.e10.

21. Kallinich T, Gattorno M, Grattan CE, de Koning HD, Traidl-Hoffmann C, Feist E, et al. Unexplained recurrent fever: when is autoinflammation the explanation? Allergy. 2013;68(3):285-96.

22. de Valles-lbáñez G, Esteve-Solé A, Piquer M, González-Navarro EA, Hernandez-Rodriguez J, Laayouni $\mathrm{H}$, et al. Evaluating the Genetics of Common Variable Immunodeficiency: Monogenetic Model and Beyond. Front. Immunol. 2018;9(636).

23. Sarmiento JD, Villada F, Orrego JC, Franco JL, Trujillo-Vargas CM. Adverse events following immunization in patients with primary immunodeficiencies. Vaccine. 2016;34(13):1611-6.

24. Sohani M, Habibi S, Delavari S, Shahkarami S, Yazdani R, Shirmast P, et al. Evaluation of patients with primary immunodeficiency associated with Bacille Calmette-Guerin (BCG)-vaccine-derived complications. Allergol Immunopathol (Madr). 2020;48(6):729-37.

25. Marciano BE, Huang CY, Joshi G, Rezaei N, Carvalho BC, Allwood Z, et al. BCG vaccination in patients with severe combined immunodeficiency: complications, risks, and vaccination policies. J. Allergy Clin. Immunol. 2014;133(4):1134-41.

26. Colditz GA, Brewer TF, Berkey CS, Wilson ME, Burdick E, Fineberg HV, et al. Efficacy of BCG vaccine in the prevention of tuberculosis. Meta-analysis of the published literature. JAMA. 1994;271(9):698702.

27. Ohmori M, Ishikawa N, Yoshiyama T, Uchimura K, Aoki M, Mori T. Current epidemiological trend of tuberculosis in Japan. Int. J. Tuberc. Lung Dis. 2002;6(5):415-23.

28. Hagiya H, Koyama T, Zamami Y, Minato $Y$, Tatebe $Y$, Mikami N, et al. Trends in incidence and mortality of tuberculosis in Japan: a population-based study, 1997-2016. Epidemiol. Infect. 2018;147:e38. 
29. Puck JM. Newborn screening for severe combined immunodeficiency and T-cell lymphopenia. Immunol. Rev. 2019;287(1):241-52.

30. Rechavi E, Lev A, Simon AJ, Stauber T, Daas S, Saraf-Levy T, et al. First Year of Israeli Newborn Screening for Severe Combined Immunodeficiency-Clinical Achievements and Insights. Front. Immunol. 2017;8:1448.

31. Argudo-Ramírez A, Martín-Nalda A, Marín-Soria JL, López-Galera RM, Pajares-García S, González de Aledo-Castillo JM, et al. First Universal Newborn Screening Program for Severe Combined Immunodeficiency in Europe. Two-Years' Experience in Catalonia (Spain). Front. Immunol. 2019;10:2406.

32. Muramatsu H, Kojima D, Okuno Y, Kataoka S, Nakajima Y, Ito T, et al. Combination of TREC Measurement and Next-Generation Sequencing in Newborn Screening for Severe Combined Immunodeficiency: A Pilot Program in Japan. Blood. 2018;132(Supplement 1):3717-.

33. Collins CJ, Yi F, Dayuha R, Whiteaker JR, Ochs HD, Freeman A, et al. Multiplexed Proteomic Analysis for Diagnosis and Screening of Five Primary Immunodeficiency Disorders From Dried Blood Spots. Front. Immunol. 2020;11(464).

34. Boisson-Dupuis S, Bustamante J, El-Baghdadi J, Camcioglu Y, Parvaneh N, El Azbaoui S, et al. Inherited and acquired immunodeficiencies underlying tuberculosis in childhood. Immunol. Rev. 2015;264(1):103-20.

35. Kulkarni M, Desai M, Gupta M, Dalvi A, Taur P, Terrance A, et al. Clinical, Immunological, and Molecular Findings of Patients with p47(phox) Defect Chronic Granulomatous Disease (CGD) in Indian Families. J. Clin. Immunol. 2016;36(8):774-84.

36. Toubiana J, Okada S, Hiller J, Oleastro M, Lagos Gomez M, Aldave Becerra JC, et al. Heterozygous STAT1 gain-of-function mutations underlie an unexpectedly broad clinical phenotype. Blood. 2016;127(25):3154.

37. Lim CK, Abolhassani H, Appelberg SK, Sundin M, Hammarström L. IL2RG hypomorphic mutation: identification of a novel pathogenic mutation in exon 8 and a review of the literature. Allergy Asthma Clin. Immunol. 2019;15:2.

38. Shearer WT, Fleisher TA, Buckley RH, Ballas Z, Ballow M, Blaese RM, et al. Recommendations for live viral and bacterial vaccines in immunodeficient patients and their close contacts. J. Allergy Clin. Immunol. 2014;133(4):961-6.

\section{Tables}

Table 1: Results of the primary survey 


\begin{tabular}{|c|c|c|c|c|c|c|}
\hline Department & $\begin{array}{l}\text { Departments } \\
\text { (total) }\end{array}$ & $\begin{array}{l}\text { Departments } \\
\text { Selected }\end{array}$ & $\begin{array}{l}\text { Selection } \\
\text { rate }(\%)\end{array}$ & $\begin{array}{l}\text { Response } \\
\text { rate (\%) }\end{array}$ & $\begin{array}{l}\text { Reported } \\
\text { Patients }\end{array}$ & $\begin{array}{l}\text { Patients } \\
\text { estimated in } \\
\text { Japan (95\% } \\
\text { Cl) }\end{array}$ \\
\hline Pediatrics & 2053 & 471 & 22.9 & 54.8 & 1011 & $\begin{array}{l}1491.8 \\
(1258.0- \\
1606.3)\end{array}$ \\
\hline $\begin{array}{l}\text { Internal } \\
\text { medicine }\end{array}$ & 6387 & 1151 & 18 & 18 & 47 & $\begin{array}{l}278.4 \\
(107.5- \\
362.1)\end{array}$ \\
\hline Hematology & 442 & 136 & 30.8 & 23.5 & 38 & $\begin{array}{l}274.2 \\
(136.3- \\
341.8)\end{array}$ \\
\hline Rheumatology & 1134 & 248 & 21.9 & 27.8 & 174 & $\begin{array}{l}665.1 \\
(343.0- \\
822.9)\end{array}$ \\
\hline Dermatology & 2501 & 485 & 19.4 & 28.9 & 37 & $\begin{array}{l}84.6(15.3- \\
118.5)\end{array}$ \\
\hline Total & 12517 & 2491 & 19.9 & 28.3 & 1307 & $\begin{array}{l}2794.2 \\
(2334.3- \\
3019.4)\end{array}$ \\
\hline
\end{tabular}

Table 2 Reported number and diagnosis of IEI patients. 

IUIS Disease
n genetic category

1.Immunodeficiencies affecting cellular and humoral immunity $(n=52)$

T-B+ severe combined immunodeficiency (SCID)

\begin{tabular}{lcc} 
YC deficiency & 26 & IL2RG \\
\hline JAK3 deficiency & 1 & JAK3 \\
\hline IL-7Ra deficiency & 1 & IL7R
\end{tabular}

T-B- SCID

\begin{tabular}{lll} 
RAG1 deficiency & 2 & RAG1 \\
\hline RAG2 deficiency & 1 & RAG2 \\
\hline Artemis deficiency & 3 & DCLRE1C \\
\hline LIG4 deficiency & 1 & LIG4 \\
\hline ADA deficiency & 3 & ADA \\
\hline SCID, undetermined & 1 & (nd)
\end{tabular}

Combined immunodeficiencies less profound than SCID

\begin{tabular}{lll} 
CD40 ligand deficiency & 9 & CD40LG \\
\hline DOCK8 deficiency & 1 & DOCK8 \\
\hline IKBKB deficiency & 1 & IKBKB \\
\hline CID, undetermined & 2 & (nd)
\end{tabular}

2. Combined immunodeficiencies with associated or syndromic features $(n=95)$

Immunodeficiency with congenital thrombocytopenia

Wiskott-Aldrich syndrome (WAS)/X linked thrombocytopenia

WAS, undetermined

$3 \quad(n d)$

DNA repair defects

\begin{tabular}{llll} 
Ataxia telangiectasia (AT) & 4 & ATM \\
\hline AT, undetermined & 2 & (nd) \\
\hline $\begin{array}{l}\text { Immunodeficiency with centromeric instability and facial } \\
\text { anomalies (ICF1) }\end{array}$ & 1 & DNMT3B
\end{tabular}

Thymic defects with additional congenital anomalies

DiGeorge syndrome 
DiGeorge syndrome, undetermined

Hyper IgE syndromes (HIES)

STAT3 deficiency (Job syndrome, AD-HIES)

other

Dyskeratosis congenita (DKC)

$\mathrm{XL}-\mathrm{DKC}$

AR-DKC due to RTEL1 deficiency

SAMD9L deficiency

Anhidrotic ectodermal dysplasia with immunodeficiency (EDA-ID)

NEMO (IKBKG) deficiency

EDA-ID, undetermined

Other defects

Kabuki syndrome 1

3.Predominantly antibody deficiencies $(n=183)$

Agammaglobulinemia

BTK deficiency (XLA)

$\mathrm{XLA}$, undetermined

CVID phenotype

APDS1 (PIK3CD GOF)

APDS2 (PIK3R1 LOF)

CD19 deficiency

NFKB1 deficiency

NFKB2 deficiency

IKAROS deficiency

others

CVID, undetermined

hyper IgM syndrome (HIMS)

AID deficiency
3 (nd)

$37 \quad$ STAT3

1 other

$1 \quad$ DKC1

1 RTEL1

1 SAMD9L

6 NEMO

3 (nd)

$1 \mathrm{KMT2D}$

57 BTK

5 (nd)

1 PIK3CD

2 PIK3RI

$1 \quad$ CD19

$3 \quad$ NFKB1

$2 \quad$ NFKB2

$3 \quad$ IKZF1

2 FANCE, RAD50

59 (nd) 
isotype, light chain, or functional deficiencies with generally normal number of B cells

\begin{tabular}{lll} 
Isolated IgG subclass deficiency & 12 & (nd) \\
\hline Selective IgA deficiency (SID) & 1 & TACl \\
SID, undetermined & 12 & (nd) \\
\hline Transient hypogammaglobulinemia of infancy & 20 & (nd)
\end{tabular}

4.Diseases of immune dysregulation $(n=47)$

Familial hemophagocytic lymphohistiocytosis (FHL) syndromes

FHL3

FHL2

FHL syndromes with hypopigmentation

Chediak-Higashi Syndrome (CHS)

Hermansky-Pudlak syndrome type 2

Regulatory $\mathrm{T}$ cell defects

IPEX

CTLA4 deficiency (ALPSV)

STAT3 GOF mutation

Autoimmunity with/without lymphoproliferation

ALPS-FAS

ALPS, undetermined

Immune dysregulation with colitis

IL-10Ra deficiency

Susceptibility to EBV and lymphoproliferative conditions

\begin{tabular}{lll} 
SH2D1A deficiency (XLP1) & 3 & SH2D1A \\
\hline XIAP deficiency (XLP2) & 14 & XIAP
\end{tabular}

5.Congenital defects of phagocyte number or function $(n=121)$

Congenital neutropenias

$\begin{array}{lll}\text { Elastase deficiency (SCN1) } & 26 & \text { ELANE } \\ \text { HAX1 deficiency (Kostmann disease) } & 2 & \text { HAX1 } \\ \text { SRP54 deficiency } & 2 & \text { SRP54 }\end{array}$

Page $14 / 21$
2 UNC13D

$4 \quad$ PRF1

$4 \quad$ LYST

$1 \quad$ AP3B1

$4 \quad$ FOXP3

$5 \quad$ CTLA4

1 STAT3

$7 \quad$ TNFRSF6

$1 \quad(n d)$

1 IL10RA 
Defects of motility

Shwachman-Diamond syndrome

Specific granule deficiency

Defects of respiratory burst

X-CGD, gp91phox

AR-CGD, p22phox

AR-CGD, p67phox

AR-CGD, p47phox

CGD, undetermined

Other non-lymphoid defects

GATA2 deficiency (MonoMac syndrome)
$3 \quad$ SBDS

1 CEBPE

66 CYBB

2 CYBA

$5 \quad$ NCF2

$1 \quad$ NCF1

$6 \quad$ (nd)

1 GATA2

6.Defects in intrinsic and innate immunity $(n=25)$

Mendelian susceptibility to mycobacterial disease (MSMD)

IFN-y receptor1 deficiency

$4 \quad$ IFNGR1

STAT1 deficiency

6 STAT1

Tyk2 deficiency

1 TYK2

Predisposition to severe viral infection

STAT1 deficiency (AR LOF)

$1 \quad$ STAT1

Predisposition to invasive fungal diseases

CARD9 deficiency

$3 \quad$ CARD 9

Predisposition to mucocutaneous candidiasis (CMC)

STAT1 GOF

8 STAT1

TLR signaling pathway deficiency with bacterial susceptibility

IRAK-4 deficiency

$1 \quad$ IRAK4

Other

$1 \quad(n d)$

7.Autoinflammatory disorders $(n=191)$

Type 1 interferonopathies

Aicardi-Goutieres syndrome

$1 \quad$ TREX1 
SAMHD1 deficiency

STING-associated vasculopathy, infantile-onset

Defects affecting the inflammasome

Familial Mediterranean fever (FMF)

FMF, undetermined

Mevalonate kinase deficiency (Hyper IgD syndrome)

Hyper IgD syndrome, undetermined

Cryopyrin associated periodic syndrome (CAPS)

Familial cold autoinflammatory syndrome (FCAS) 2

FCAS 4

CAPS, undetermined

Non-inflammasome-related conditions

TNF receptor-associated periodic syndrome (TRAPS)

TRAPS, undetermined

Blau syndrome

Chronic recurrent multifocal osteomyelitis (CRMO)

Deficiency of IL-36 receptor antagonist (DITRA)

CARD14 mediated psoriasis

Otulipenia/ORAS

A20 haploinsufficiency

ADA2 deficiency (DADA2)
1 SAMHD 1

1 TMEM173
90 MEFV

$31 \quad$ (nd)

$1 \quad \mathrm{MVK}$

1 (nd)

$21 \quad$ NLRP3

$1 \quad$ NLRP12

$1 \quad$ NLRC4

4 (nd)

8 TNFRSF1A

1 (nd)

3 (nd)

2 (nd)

2 TL36RN

2 CARD14

1 OTULIN

17 TNFAIP3

$2 \quad \mathrm{ADA} 2$

8. Complement deficiencies $(n=34)$

Hereditary angioedema (HAE)

C1 inhibitor deficiency

14 SERPING1

$\mathrm{HAE}$, undetermined

16 (nd)

Cq1 deficiency

1 C1QB

C7 deficiency

1 (nd)

C9 deficiency

$2 \quad \mathrm{Cg}$

9.Phenocopies of IEI $(n=2)$ 
nd: not determined, CVID: common variable immunodeficiency, APDS: activated PI3K delta syndrome, GOF: gain of function, IPEX: immuno dysregulation, polyendocrinopathy, entropathy, X-linked, ALPS: autoimmune lymphoproliferative syndrome, SCN: severe congenital neutropenia, CGD: chronic granulomatous disease, X-: X-linked, AR-: autosomal recessive, LOF: loss-of-function

Table 3. BCG infections from the whole survey

\begin{tabular}{ll} 
disease & $n$ \\
\hline WAS/XLT & 2 \\
\hline EDA-ID & 1 \\
\hline CVID & 1 \\
\hline XLP & 1 \\
\hline CGD & 20 \\
\hline MSMD & 6 \\
\hline STAT1 deficiency (AR-LOF) & 1 \\
\hline
\end{tabular}

WAS: Wiskott-Aldrich syndrome, XLT: X-linked thrombocytopenia, EDA-ID: Anhidrotic ectodermal dysplasia with immunodeficiency, CVID: Common variable immunodeficiency, XLP: X-linked lymphoproliferative disease, CGD: Chronic granulomatous disease, MSMD: Mendelian susceptibility to mycobacterial disease, AR-LOF: Autosomal recessive loss-of-function

\section{Figures}




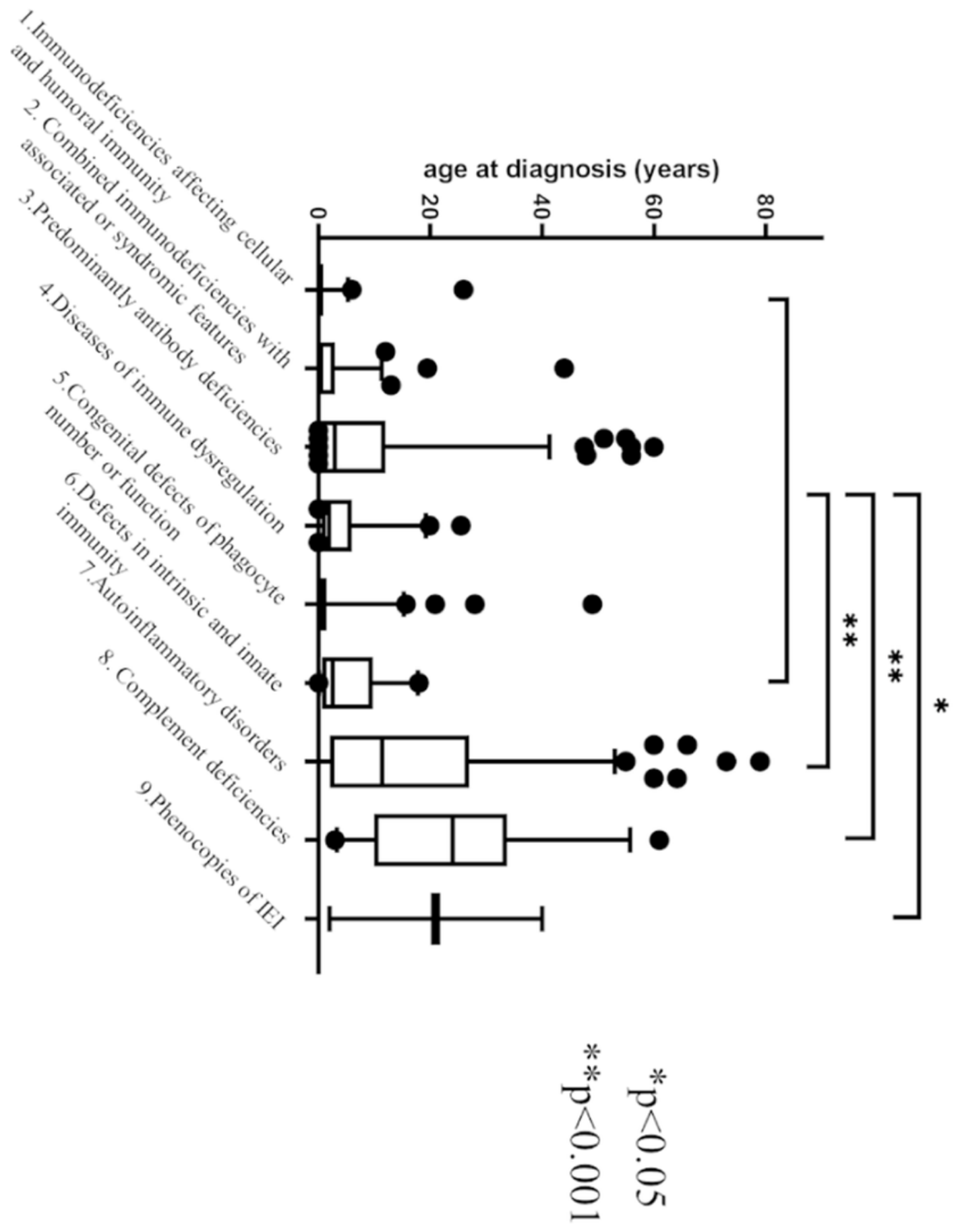

\section{Figure 1}

The median age at diagnosis for each IUIS category. The box portion represents the 25th and 75th percentile, whiskers represent the 5th and 95th percentile. The median diagnostic age for autoinflammatory disorders and complement deficiencies was significantly older than other groups $(p<0.001)$ 
$\underline{\text { IUIS category }}$

1. Defects of cellular and humoral immunity

HIES (STAT3 LOF)

2. CID with syndromic $(n=36)$ features

EDA-ID (n=9)

3. Predominantly antibody deficiency

XLA $(n=62)$

4. Diseases of immune dysregulation

5. Congenital defects of phagocytes

6. Defects in intrinsic and innate immunity

disease

SCID $(n=39)$ $\operatorname{MSMD}(\mathrm{n}=11)$

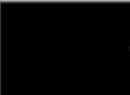

$\begin{array}{lll}9 & 1 & 6\end{array}$

23
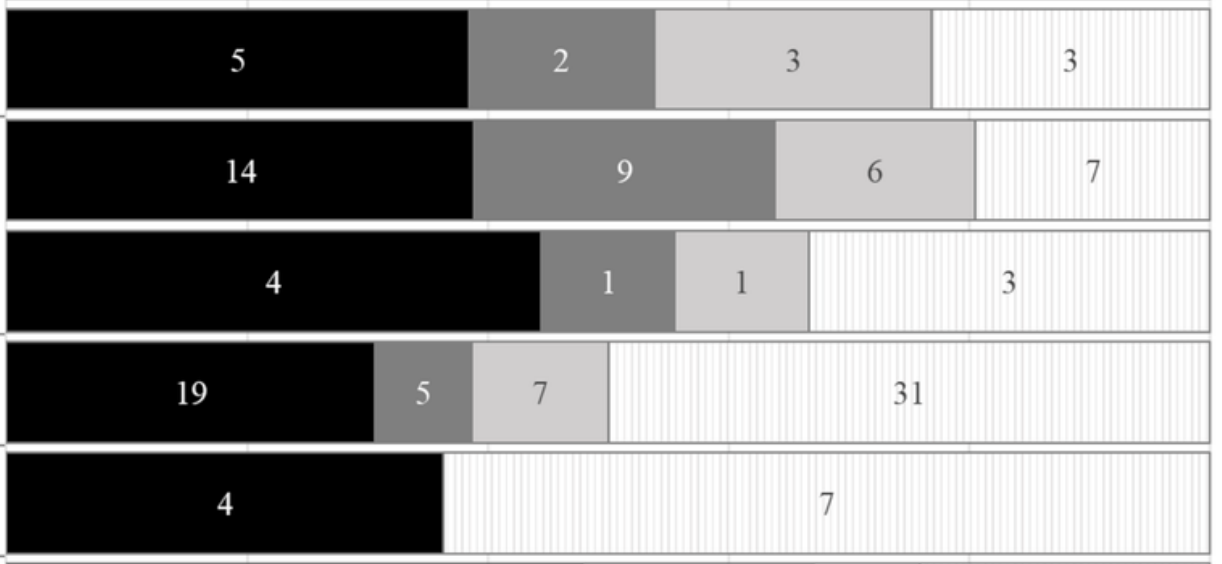

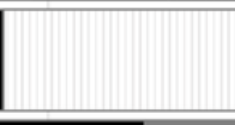

38

15

7
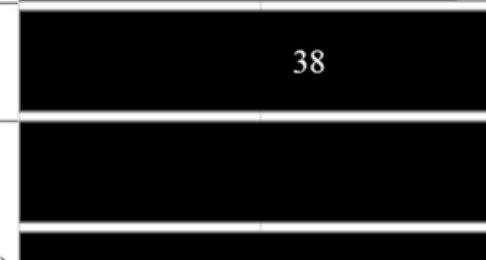

4

9

2

2

19

$(\mathrm{n}=8)$

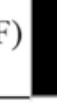

$0 \%$

$20 \%$

$40 \%$

$80 \%$

any history of BCG $\square$ history of live vaccine but no BCG $\square$ history of inactivated vaccines only $\square$ nd or no vaccines

\section{Figure 2}

The vaccination status of patients in representative disease groups. Patients were assigned to either of the following; 1 ) patients who received $B C G, 2$ ) patients with a positive history of a live vaccine but no $\mathrm{BCG}$, or 3) patients who received inactivated vaccines only. The percentage of each group is shown by bar graphs, the actual number of patients for each group is labeled inside the bars. 
disease

Total $(n=195)$

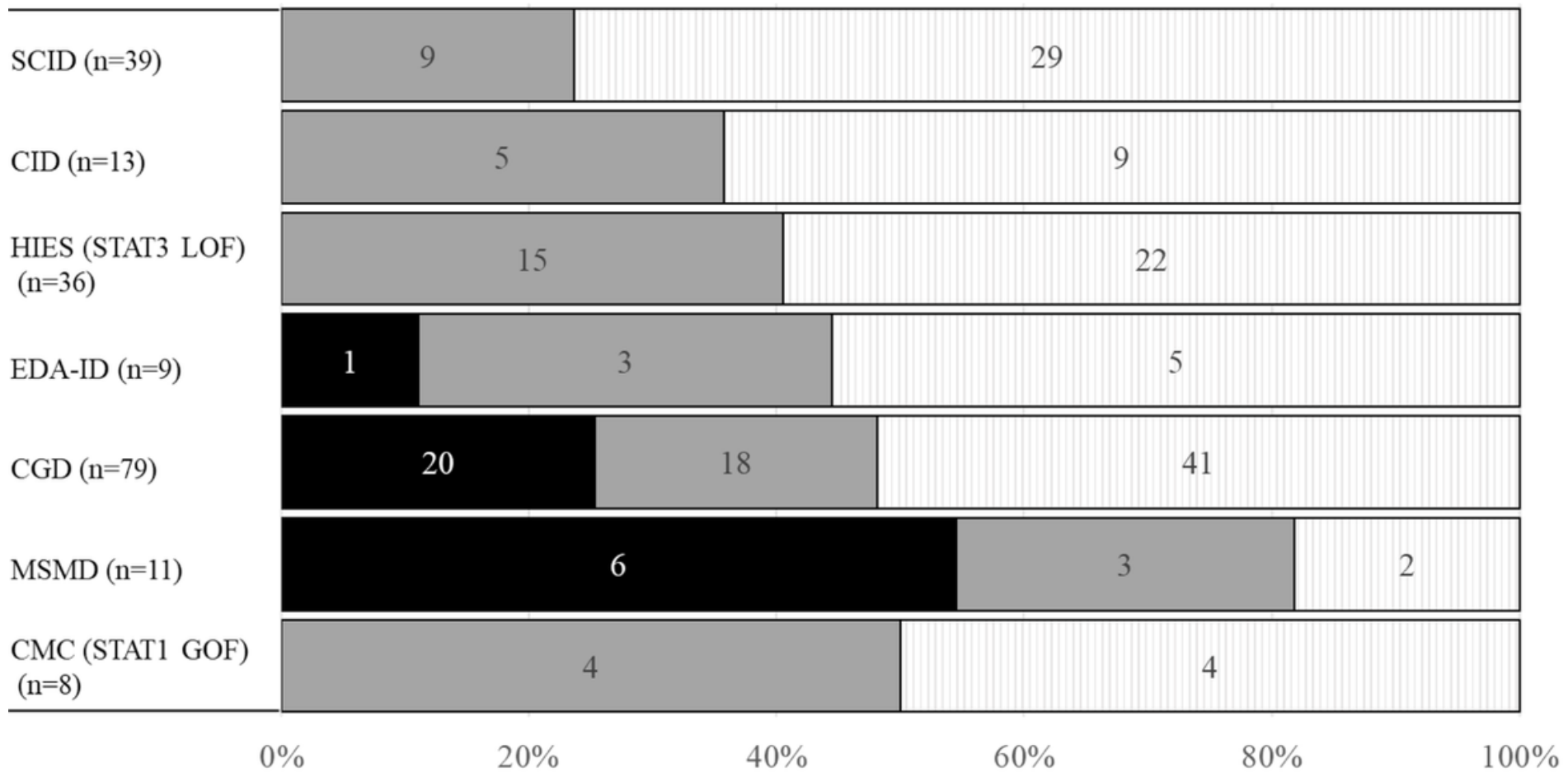

$0 \%$

$20 \%$

$40 \%$

$60 \%$

$\square$ did not receive BCG

\section{Figure 3}

The rate of BCG administration and BCG infections among diseases for which BCG is contraindicated. Patients were assigned to either of the following; 1) patients who received BCG and developed a BCG infection, 2) patients who received BCG but did not have a reported BCG infection, or 3) patients who did not receive BCG. The percentage of each group is shown by bar graphs, the actual number of patients for each group is labeled inside the bars. 


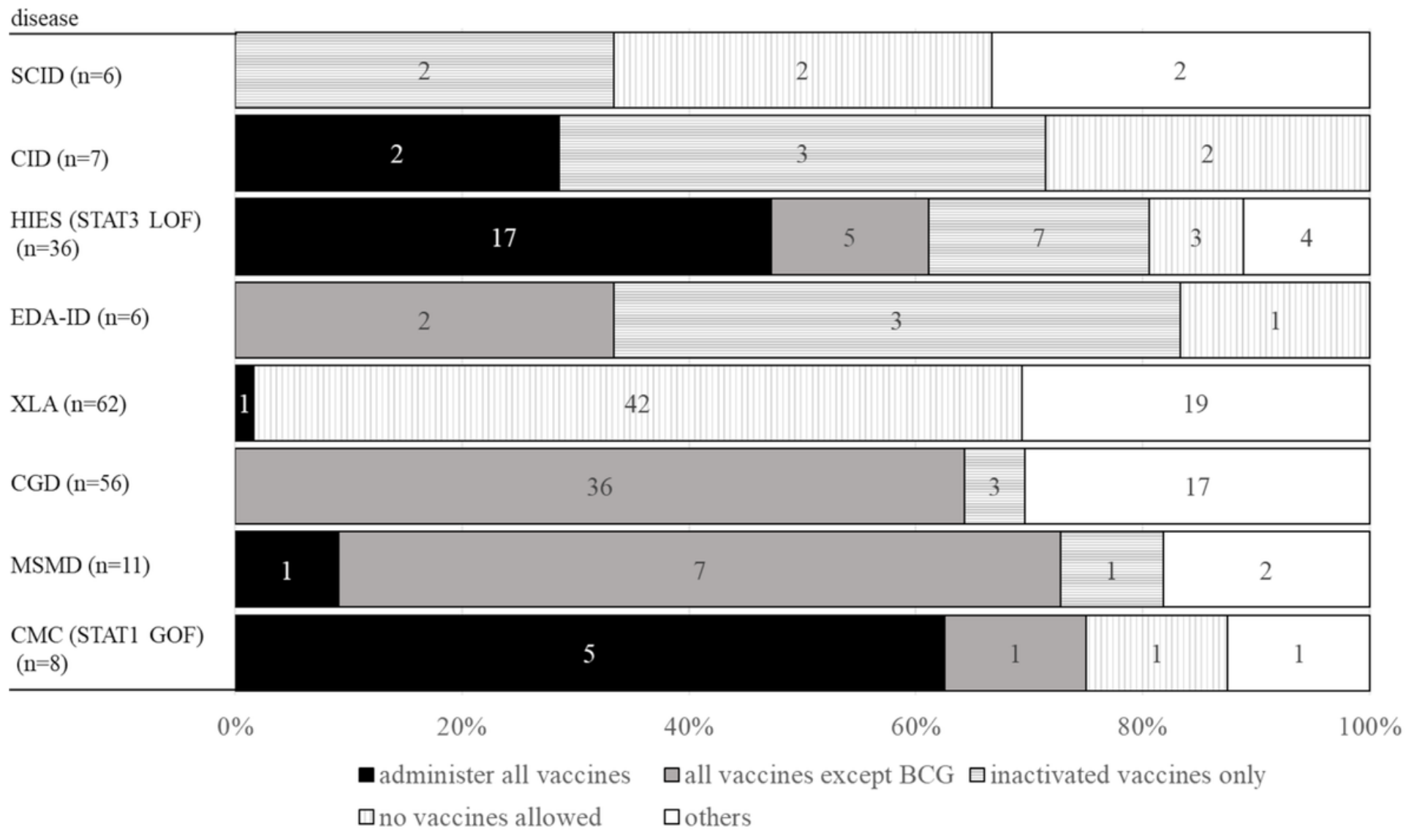

\section{Figure 4}

The vaccination policy of attending physicians for representative diseases, patients who received SCT are excluded. Physicians were asked whether their policy was to 1 ) administer all vaccines, 2) administer all vaccines except BCG, 3) administer inactivated vaccines only, or 4) administer no vaccines. The percentage of each group is shown by bar graphs, the actual number of cases for each group is labeled inside the bars.

\section{Supplementary Files}

This is a list of supplementary files associated with this preprint. Click to download.

- FigureS1.pdf

- TableS1.pdf 